\title{
Depressive Symptoms And Stigmatization Among Parents Of Children With Intellectual Disabilities: Cross-Sectional Survey In Public Primary Special Schools In Nairobi, Kenya
}

\author{
Mercy Githara \\ University of Nairobi \\ Anne Obondo \\ University of Nairobi \\ Albert Tele \\ CRID Consultants \\ Graham Thornicroft \\ King's College London School of Medical Education \\ Manasi Kumar ( $\square$ manni_3in@hotmail.com ) \\ University of Nairobi https://orcid.org/0000-0002-9773-8014
}

\section{Research}

Keywords: stigma and discrimination, intellectual disabilities, depressive symptoms

Posted Date: March 20th, 2020

DOI: https://doi.org/10.21203/rs.3.rs-17992/v1

License: (9) (1) This work is licensed under a Creative Commons Attribution 4.0 International License. Read Full License 


\section{Abstract}

Background

Parents of children with intellectual disabilities may develop depressive symptoms and are prone to stigmatization due to stressful challenges encountered when providing care for their children. The study objective was to determine the prevalence of depressive symptoms and stigmatization among parents of children with intellectual disabilities.

Method

A cross-sectional descriptive study design was used to sample 121 parents of children with intellectual disabilities in four public primary special schools in Nairobi, Kenya Data were collected using a researcher designed socio-demographic questionnaire, Beck Depression Inventory (BDI-II) to screen depressive symptoms and the DISC-12 used to determine stigmatization among parents of children with intellectual disabilities. Descriptive statistics were used to examine the depression scores, discrimination and stigma scores. Independent samples t-test, and one-way analyses of variance (ANOVA) were used to identify group differences at the bivariate level. Generalized linear models were used to identify independent predictors of discrimination and Stigma.

Results

Of the 121 parents assessed for depression $24 \%(n=29)$ of parents met the criterion for being at risk of depression using BDI scores (score of 21 and above). Stigma and discrimination were significantly related to parent's depressive symptoms, independently of other variables. On multivariate linear regression individual predictors showed that being female $(P=<0.001)$, younger age of between 18-26 $(P=<0.003)$, being divorced/ separated $(p=<0.0001)$, lower education $(P=<0.0001)$ and caregiver burden $(P=0.033)$ were predictors of depression and high levels of stigmatization.

Conclusions

The risk of depression is high among parents of children with intellectual disabilities. Results of this study suggest that interventions could be developed that have a focus on the health or well- being of parents. Reduction of societal stigma could reduce psychological distress to parents.

\section{Background}

Parenting is a process of promoting and supporting the physical, emotional social and intellectual wellbeing of an infant to adulthood mainly by the biological parents or a caregiver(Britto et al., 2017). Parent's psychological wellbeing depends on a child's health, socio-emotional, cognitive and physical development. Difficulties in functioning in these areas often lead to distress in the parenting roles that may lead to short term or long-term difficulties (Aaron Resch, Elliott, \& Benz, 2012). There are estimated 15\% of the world's population who live with disability with an estimated 93 million children of ages 0-14 years who are living with a moderate or severe disability, and most of these are in low and middle-income countries (LMICs)(WHO, 2011). Children with intellectual disability usually differ from normal child development and often their life long disability poses specific challenges to their parents or caregivers (Singer, 2006). These challenges are exacerbated when the caregivers themselves are based in LMICs or extremely low resource contexts(Charles Masulani-Mwale, Kauye, Gladstone, \& Mathanga, 2019). Such challenges can include unusual demands that include disrupted family and social relationships, exhaustion resulting from extra care required financial difficulties incurred due to treatment and other services, stigmatization from society and cultural beliefs and general parenting distress (Burke, 2003; C. Masulani-Mwale, Mathanga, Kauye, \& Gladstone, 2018). These challenges can lead to the development of depressive symptoms especially where resources and support programs are limited (Gallagher \& Hannigan, 2014).

\section{Risk Factors For Parents Of Children With Intellectual Disabilities}

Parents of children with intellectual disabilities are at risk for higher stress levels than parents of children without disabilities(Estes et al., 2013). Parental stress continues to be a centre of attention of research because it has been linked with many negative outcomes such as parental depression, marital conflict, poor physical health and ineffective parenting(Neece \& Baker, 2008; van der Mark, Conradie, Dedding, \& Broerse, 2019).

Poor understanding of behaviors manifested by children with intellectual disabilities can promote further isolation; often cause rejection by their peers, school authorities and the society at large. This can create an overwhelming responsibility for the parents causing despair, hopelessness, guilt and even helplessness, leading to depressive symptoms which are largely unrecognized(Bourke-Taylor, Pallant, Law, \& Howie, 2012; Eisenhower, Baker, \& Blacher, 2005).

Another risk of parental stress is stigma(Cantwell, Muldoon, \& Gallagher, 2015). Stigma refers to negative impacts of behaviour and attitudes from the public (E Goffman, 2009). Stigma has been defined as "an attribute that is deeply discrediting and reduces the bearer 'from whole and usual person' to a tainted discounted one (Erving Goffman, 1963)". In a revised definition of stigma by (Thornicroft, Rose, Kassam, \& Sartorius, 2007)stigma has three elements; a) problems of knowledge (ignorance or misinformation), b) problems of attitudes (prejudice), c) problems of behaviour discrimination. Individuals with intellectual disabilities (ID) have been one of the most stigmatized groups in any society. Research has shown that individuals and family caregivers of people with ID experience stigma that negatively impacts on their psychological wellbeing(Ali, Hassiotis, Strydom, \& King, 2012).

Stigma and discrimination are both rampant in the context of children with ID and the internalized (referring to feelings of shame and the anticipation of prejudice that prevents people from talking about their experiences and impedes them looking for help) and externalized stigma (evasion and experiences 
of discrimination) can usurp the family members including caregivers too(Tilahun et al., 2016). Stigma may emanate from caregivers' poor knowledge about the perceived causes and prognosis of developmental disorder in their children or from the poor literacy of the community. There can be religious attributions to these developmental disabilities(Owalabi Bakari et al., 2009).

In the developing countries like Kenya, depressive symptoms of parents of children with ID have been unrecognized due to limited public policy and research in this area. A study undertaken by (Mbugua, Kuria, \& Ndetei, 2011)on the prevalence of depression and burden of care among family caregivers of intellectually impaired children at Gachie Catholic Parish in Kiambu. This is one of the few studies from Kenya though it focused on the rural setting of Kiambu and it sought caregivers in a community church.

In addition to the limited studies that have been carried out on prevalence of depressive symptoms in parents of children with intellectual disability, anecdotal reports show that the incidence of children with ID is high suggesting the burden of care of such children could be overwhelming to parents in this country leading to depressive symptoms.

\section{Objectives}

The overall aim of this study is to establish the prevalence of depressive symptoms and stigmatization among parents of children with intellectual disabilities. The specific objectives aimed at determining the socio-demographic characteristics of parents of children with intellectual disabilities, the levels and severity of depressive symptoms among parents of children with disabilities and, finally, establishing the association between stigmatization and depressive symptoms among parents of children with intellectual disabilities.

\section{Methods}

\section{Design and study population}

We adopted a cross-sectional survey design. The study was conducted among 121 parents of children with intellectual disabilities in four public special schools in Nairobi County. Nairobi is the capital city of Kenya with six special schools closely located within proximity and easily accessible in the capital. These schools are governed by the county government under the Ministry of Education. Enrolment to these schools is done by the Kenya Institute of Education which make an education assessment and determine level of disability. During the period of the study the total number of learners registered in these schools was estimated at 690 . Majority of the learners (90\%) experienced mild to moderate intellectual disability. Ten percent of the learners had severe disability. These schools have a specified curriculum on functional academic subjects and vocational training for the mild - moderate learners while life skills and daily living skills are targeting those young people who are severely impaired.

\section{Study Participants}

Participants above the age of 18 who were willing to give informed consent were recruited. In the selection of 121 participants we used an estimated prevalence rate of $79 \%$ of caregivers with depression from(Mbugua et al., 2011). The Cochrane formula (Czaja \& Blair, 2005) was used for the sample size calculation. The formula addresses continuous and categorical statistical variables and applies key risk factors like confidence level that is acceptable (95\%) and a precision of 0.05 . In order to identify caregivers of children with intellectual impairment we decided to approach special needs schools where the parents could be contacted. This means that we required 186 parents. The total number of children in the four targeted schools is 682 . The number was adjusted for a finite population; a total of 121 participants were required. Proportionate samplings were used to enroll the number of parents in each school.

\section{Measures}

\section{Socio-demographic characteristics}

A researcher designed socio-demographic questionnaire was developed to capture the respondent's characteristics as well as their children and family. These included age, income, marital status, and employment and education status, number of children in the household. For the index child we probed about the age, gender and number of years in the residential school.

\section{Beck Depression Inventory-II (BDI-II)}

The BDI-II (Beck, Steer, \& Brown, 1996) is a 21-item scale which measures depression symptomatology. Each question on the BDI-II is scored from 0-3 with a higher number indicating greater severity. A total score is calculated as the sum of the 21 items with a range of $0-63$. The clinical cut-offs are $11-16$ (mild mood disturbance), 17-20 (borderline clinical depression), 21-30 (moderate depression), 31-40 (severe depression), and 40-63 (extreme depression). Internal consistency is high, Cronbach's a = .92 (Beck et al., 1996). The BDI-II has been used in Kenya and other countries (Ghassemzadeh, Mojtabai, Karamghadiri, \& Ebrahimkhani, 2005; Kojima et al., 2002; Musyimi et al., 2017; Ndetei et al., 2009). 


\section{Discrimination And Stigma Scale Version 12 (DISC-12)}

DISC-12 is a 34 item interview-based and standardized tool for assessment of discrimination that has been used in both high income countries and lowand-middle-income countries (Brohan et al., 2013; Thornicroft et al., 2007). DISC-12 has good psychometric properties including inter-rater reliability (weighted kappa range: $0.62-0.95)$, internal consistency $(a=0.78)$ and test-retest reliability (weighted kappa range: 0.56-0.89) (Brohan et al., 2013). It consists of a global scale and four subscales: (1) Unfair treatment (item 1-22); (2) Stopping self (item 23-26); (3) Overcoming stigma (item 27-28), and (4) Positive treatment (item 29-34). The unfair treatment subscale assesses unjust treatment by other people and higher scores indicate greater experienced discrimination. The stopping self-subscale explores the extent to which an individual limits his/her activity of daily living (e.g. work) due to fear of stigma and higher scores mean higher anticipated discrimination. The overcoming stigma subscale measures an individual's ability to overcome stigma and higher scores indicate a higher ability to cope with discrimination. The positive treatment subscale assesses positive treatment received by an individual on account of mental illness and higher scores mean greater positive treatment received by the individual. The responses to the DISC-12 are rated on a four-point Likert scale (Not at all $=0, \mathrm{~A}$ little $=1$, moderately $=2$, and A lot $=3$ ). The mean for the overall and subscales cores were calculated by summation of the rating (0-3) for each item and dividing with the total number of applicable terms. (Brohan et al., 2013). The higher the scores the greater the stigma.

A pre-test baseline assessment was made using these questionnaires was carried out on a few of the subjects to establish the reliability and validity of data collection instruments. The instruments were corrected in close collaboration with the supervisors and other professional in field. Parents attending school meetings in special schools were given verbal as well as written participants form explaining purpose of study and confidentiality was assured. Those who volunteered gave written consent to participate and were told that they could decide to stop filling in the questionnaires anytime. No financial compensation was given for their participation.

Individual questionnaires were provided and participants who required help in completing the questionnaire were assisted by the researcher who read out the questions to them without interpreting them. Parents filled the questionnaires at their own discretion and privacy was safeguarded by allowing them sit where they felt comfortable in the school halls. Completed questionnaires were collected by the researcher, stored safely before analysis was done. Psychological support was given to parents who elicited emotional distress during and after filling in the questionnaires.

\section{Ethics And Informed Consent}

The University of Nairobi/Kenyatta National Hospital Ethics Review Committee provided ethical approval for this study (P100/02/2015). All patients included in this study provided informed consent prior to participation in the study and gave consent for publication of the findings.

\section{Data Management And Statistical Analysis}

Descriptive statistics were used to examine the depression scores, discrimination and stigma scales by means of means and standard deviations for continuous variables and proportions for categorical variables. Independent samples t-test, ANOVA, were used to identify group differences. Generalized linear models were used to identify independent predictors of discrimination and stigma. All analyses were conducted using IBM SPSS version 23 (IBM, New York USA).

\section{Results}

\section{Socio Demographic Characteristics}

Of the 121 parents who participated in the study $77.69 \%(n=94)$ were female. Socio-demographic characteristics of the respondents are presented in Table 1. Parent's age ranged from 18 years to 45 years and above. More than half of the parents $72.7 \%(n=88)$ were in the age band of $36-45$ years and above. Most of the parents were married $69.9 \%(n=79)$, Most of the parents $61.1 \%(n=69)$ had post-secondary education at the time of the study were employed $78.5 \%(n=95)$, either working part time, full time or self-employed. More than half $(68.8 \% n=75)$ were earning a monthly income of more than Ksh 10,000 . Most of those employed were self-employed $38.02 \%(n=46)$. More than $3 / 4$ of our sample $(83.9 \%)$ indicated that they had experienced significant care burden. 
Table 1

Socio-Demographics and other characteristics of the respondents

\begin{tabular}{|c|c|c|c|c|c|}
\hline \multirow[t]{2}{*}{ Variable } & \multirow[t]{2}{*}{ Category } & Overall & Male & Female & \multirow[t]{2}{*}{ P-Value } \\
\hline & & $N=121$ & $\mathrm{n}=27$ & $\mathrm{n}=94$ & \\
\hline \multirow[t]{2}{*}{ Respondent's Gender } & Male & $27(22.3 \%)$ & - & - & \\
\hline & Female & $94(77.7 \%)$ & - & - & \\
\hline \multirow[t]{4}{*}{ Respondents' Age } & $18-25 y r s$ & $18(14.9 \%)$ & $7(38.9 \%)$ & $11(61.1 \%)$ & \multirow[t]{4}{*}{$0.133^{\star}$} \\
\hline & $26-35 y r s$ & $15(12.4 \%)$ & $4(26.7 \%)$ & $11(73.3 \%)$ & \\
\hline & $36-45 y r s$ & $41(33.9 \%)$ & $10(24.4 \%)$ & $31(75.6 \%)$ & \\
\hline & $45+$ & $47(38.8 \%)$ & $6(12.8 \%)$ & $41(87.2 \%)$ & \\
\hline \multirow[t]{4}{*}{ Marital Status } & Single & $17(15.0 \%)$ & $3(17.6 \%)$ & $14(82.4 \%)$ & \multirow[t]{4}{*}{$0.691^{\star}$} \\
\hline & Married & $79(69.9 \%)$ & $17(21.5 \%)$ & $62(78.5 \%)$ & \\
\hline & Divorced/Separated & $17(15.0 \%)$ & $5(29.4 \%)$ & $12(70.6 \%)$ & \\
\hline & Missing & 8 & & & \\
\hline \multirow[t]{2}{*}{ Employment Status } & Unemployed & $26(21.5 \%)$ & $2(7.7 \%)$ & $24(92.3 \%)$ & \multirow[t]{2}{*}{0.043} \\
\hline & Employed & $95(78.5 \%)$ & $25(26.3 \%)$ & $70(73.7 \%)$ & \\
\hline \multirow[t]{3}{*}{ Income } & 10,000 and Below & $34(31.2 \%)$ & $5(14.7 \%)$ & $29(85.3 \%)$ & \multirow[t]{3}{*}{0.169} \\
\hline & Above 10,000 & $75(68.8 \%)$ & $20(26.7 \%)$ & $55(73.3 \%)$ & \\
\hline & Missing & 12 & & & \\
\hline \multirow[t]{4}{*}{ Education Level } & Primary and Below & $13(11.5 \%)$ & $3(23.1 \%)$ & $10(76.9 \%)$ & \multirow[t]{4}{*}{0.825} \\
\hline & Secondary & $31(27.4 \%)$ & $8(25.8 \%)$ & $23(74.2 \%)$ & \\
\hline & Post-Secondary & $69(61.1 \%)$ & $14(20.3 \%)$ & $55(79.7 \%)$ & \\
\hline & Missing & 8 & & & \\
\hline \multirow[t]{3}{*}{ Number of Children } & 3 and below & $84(72.4 \%)$ & $20(23.8 \%)$ & $64(76.2 \%)$ & \multirow[t]{3}{*}{0.338} \\
\hline & Above 3 & $32(27.6 \%)$ & $5(15.6 \%)$ & $27(84.4 \%)$ & \\
\hline & Missing & 5 & & & \\
\hline \multirow[t]{5}{*}{ Age of Children) with Intellectual Disability } & 9 and Below & $17(14.5 \%)$ & $2(11.8 \%)$ & $15(88.2 \%)$ & \multirow[t]{5}{*}{0.203} \\
\hline & $10-12$ Years & $35(29.9 \%)$ & $7(20.0 \%)$ & $28(80.0 \%)$ & \\
\hline & $13-18$ Years & $26(22.2 \%)$ & $9(34.6 \%)$ & $17(65.4 \%)$ & \\
\hline & Over 18 Years & $39(33.3 \%)$ & $6(15.4 \%)$ & $33(84.6 \%)$ & \\
\hline & Missing & 4 & & & \\
\hline \multirow[t]{4}{*}{ Number of People Living With } & $1-3$ & $38(32.8 \%)$ & $8(21.1 \%)$ & $30(78.9 \%)$ & \multirow[t]{4}{*}{0.854} \\
\hline & $4-6$ & $64(55.2 \%)$ & $13(20.3 \%)$ & $51(79.7 \%)$ & \\
\hline & $6+$ & $14(12.1 \%)$ & $2(14.3 \%)$ & $12(85.7 \%)$ & \\
\hline & Missing & 5 & & & \\
\hline \multirow[t]{3}{*}{ Care Burden } & Yes & $99(83.9 \%)$ & $20(20.2 \%)$ & $79(79.8 \%)$ & \multirow[t]{3}{*}{$0.114^{\star}$} \\
\hline & No & $19(16.1 \%)$ & $7(36.8 \%)$ & $12(63.2 \%)$ & \\
\hline & Missing & 3 & & & \\
\hline
\end{tabular}

Table 2 presents the description of the DISC-12 sub-scales. The mean scores of unfair treatment was $0.9(S D=0.6$, range of 0-2.6) stopping self, mean $=$ $0.5(S D=0.5$, range of $0-2.3)$, range 0 to 2.3 , overcoming stigma mean $=1.2(S D=0.8$, range of $0-3)$ and positive treatment mean $=1.7(S D=0.9$ range of $0-3)$.

Experienced And Anticipated Discrimination (DISC) And Depression (BDI) 
Table 2

Stigma (DISC) Scores

\begin{tabular}{|c|c|c|c|c|c|c|c|c|}
\hline \multirow{2}{*}{$\begin{array}{l}\text { Disc } 12 \text { Scales } \\
\text { subscales }\end{array}$} & \multicolumn{4}{|c|}{ Mean Scores } & \multicolumn{4}{|l|}{ Total Score } \\
\hline & $\begin{array}{l}\text { Mean } \\
(95 \% \text { C.I) }\end{array}$ & Median & S.D & Range & $\begin{array}{l}\text { Mean } \\
(95 \% \text { C.I) }\end{array}$ & Median & S.D & Range \\
\hline 1. Unfair Treatment & $\begin{array}{l}0.9 \\
(0.7 \text { to } 1.0)\end{array}$ & 0.8 & 0.6 & 0.0 to 2.6 & $\begin{array}{l}10.1 \\
\text { (9.0 to } 11.2)\end{array}$ & 9.0 & 6.0 & 0.0 to 21.0 \\
\hline 2. Stopping Self & $\begin{array}{l}0.5 \\
(0.4 \text { to } 0.6)\end{array}$ & 0.5 & 0.5 & 0.0 to 2.3 & $\begin{array}{l}1.3 \\
(1.1 \text { to } 1.5)\end{array}$ & 1.0 & 1.2 & 0.0 to 4.0 \\
\hline 3. Overcoming stigma & $\begin{array}{l}1.2 \\
(1.0 \text { to } 1.3)\end{array}$ & 1.5 & 0.8 & 0.0 to 3.0 & $\begin{array}{l}1.0 \\
(0.8 \text { to } 1.1)\end{array}$ & 1.0 & 0.7 & 0.0 to 2.0 \\
\hline 4. Positive treatment & $\begin{array}{l}1.7 \\
(1.6 \text { to } 1.9)\end{array}$ & 1.8 & 0.9 & 0.0 to 3.0 & $\begin{array}{l}3.9 \\
\text { (3.6 to } 4.2 \text { ) }\end{array}$ & 5.0 & 1.5 & 0.0 to 5.0 \\
\hline BDI-II Depression & - & - & - & - & $\begin{array}{l}11.5 \\
(10.0-13.1)\end{array}$ & 9.0 & 8.5 & 1 to 39 \\
\hline
\end{tabular}

\section{Prevalence Of Depression}

Our results demonstrate that $41.3 \%$ of the participants were clinically at risk of depression as assessed using BDI. These results show that the parental BDI scores indicate presence of mild depression to severe depression in the caregivers. Mean depression score was 11.5 (SD = 8.5, range of $1-39)$.

\section{Bivariate Associations And Multivariate Analysis}

The bivariate association between stigma, the socio-demographic and other characteristics are presented in Table 3. Significant associations are those which were $\mathrm{P}<0.05$ and these are indicated by bolded values for each of the four subscales. 
Table 3

Bivariate analysis of factors associated with Stigma

\begin{tabular}{|c|c|c|c|c|c|c|c|c|c|c|c|c|c|}
\hline \multirow[t]{2}{*}{ Variable } & \multirow[t]{2}{*}{ Category } & \multicolumn{3}{|c|}{ Unfair treatment } & \multicolumn{3}{|c|}{ Stopping self } & \multicolumn{3}{|c|}{ Overcoming stigma } & \multicolumn{3}{|c|}{ Positive treatment } \\
\hline & & $\mathrm{N}$ & $\begin{array}{l}M \pm \\
S D\end{array}$ & Sig. & $\mathrm{N}$ & $\begin{array}{l}M \pm \\
S D\end{array}$ & Sig. & $\mathrm{N}$ & $\begin{array}{l}M \pm \\
S D\end{array}$ & Sig. & $\mathrm{N}$ & $\begin{array}{l}M \pm \\
S D\end{array}$ & Sig. \\
\hline \multirow[t]{2}{*}{ Respondent's Gender } & Male & 26 & $\begin{array}{l}0.91 \pm \\
0.7\end{array}$ & \multirow[t]{2}{*}{0.666} & 24 & $\begin{array}{l}0.52 \\
\pm 0.5\end{array}$ & \multirow[t]{2}{*}{0.927} & 24 & $\begin{array}{l}1.25 \\
\pm 0.8\end{array}$ & \multirow[t]{2}{*}{0.534} & 24 & $\begin{array}{l}1.80 \\
\pm 0.8\end{array}$ & \multirow[t]{2}{*}{0.752} \\
\hline & Female & 91 & $\begin{array}{l}0.85 \pm \\
0.6\end{array}$ & & 89 & $\begin{array}{l}0.53 \\
\pm 0.6\end{array}$ & & 85 & $\begin{array}{l}1.14 \\
\pm 0.8\end{array}$ & & 89 & $\begin{array}{l}1.73 \\
\pm 0.8\end{array}$ & \\
\hline \multirow[t]{4}{*}{ Respondents' Age } & $18-25 y r s$ & 17 & $\begin{array}{l}0.99 \pm \\
0.8\end{array}$ & \multirow[t]{4}{*}{0.262} & 16 & $\begin{array}{l}0.55 \\
\pm 0.3\end{array}$ & \multirow[t]{4}{*}{0.180} & 16 & $\begin{array}{l}1.41 \\
\pm 0.7\end{array}$ & \multirow[t]{4}{*}{0.058} & 16 & $\begin{array}{l}1.24 \\
\pm 0.7\end{array}$ & \multirow[t]{4}{*}{0.001} \\
\hline & $26-35 y r s$ & 15 & $\begin{array}{l}0.58 \pm \\
0.4\end{array}$ & & 13 & $\begin{array}{l}0.21 \\
\pm 0.4\end{array}$ & & 12 & $\begin{array}{l}0.92 \\
\pm 0.9\end{array}$ & & 13 & $\begin{array}{l}1.90 \\
\pm 0.9\end{array}$ & \\
\hline & $36-45 y r s$ & 39 & $\begin{array}{l}0.92 \pm \\
0.7\end{array}$ & & 39 & $\begin{array}{l}0.57 \\
\pm 0.7\end{array}$ & & 39 & $\begin{array}{l}1.35 \\
\pm 0.9\end{array}$ & & 39 & $\begin{array}{l}2.11 \\
\pm 0.9\end{array}$ & \\
\hline & $45+$ & 46 & $\begin{array}{l}0.86 \pm \\
0.6\end{array}$ & & 45 & $\begin{array}{l}0.57 \\
\pm 0.5\end{array}$ & & 42 & $\begin{array}{l}0.96 \\
\pm 0.7\end{array}$ & & 45 & $\begin{array}{l}1.57 \\
\pm 0.7\end{array}$ & \\
\hline \multirow[t]{3}{*}{ Marital Status } & Single & 17 & $\begin{array}{l}0.78 \pm \\
0.5\end{array}$ & \multirow[t]{3}{*}{0.000} & 13 & $\begin{array}{l}0.69 \\
\pm 0.5\end{array}$ & \multirow[t]{3}{*}{0.031} & 12 & $\begin{array}{l}1.17 \\
\pm 0.6\end{array}$ & \multirow[t]{3}{*}{0.003} & 15 & $\begin{array}{l}1.65 \\
\pm 0.6\end{array}$ & \multirow[t]{3}{*}{0.597} \\
\hline & Married & 76 & $\begin{array}{l}0.79 \pm \\
0.6\end{array}$ & & 77 & $\begin{array}{l}0.44 \\
\pm 0.5\end{array}$ & & 74 & $\begin{array}{l}1.05 \\
\pm 0.8\end{array}$ & & 75 & $\begin{array}{l}1.79 \\
\pm 0.8\end{array}$ & \\
\hline & Divorced/Separated & 17 & $\begin{array}{l}1.45 \pm \\
0.7\end{array}$ & & 17 & $\begin{array}{l}0.77 \\
\pm 0.5\end{array}$ & & 17 & $\begin{array}{l}1.76 \\
\pm 0.5\end{array}$ & & 17 & $\begin{array}{l}1.95 \\
\pm 0.5\end{array}$ & \\
\hline \multirow[t]{2}{*}{ Employment Status } & Unemployed & 25 & $\begin{array}{l}0.55 \pm \\
0.6\end{array}$ & \multirow[t]{2}{*}{0.005} & 25 & $\begin{array}{l}0.57 \\
\pm 0.6\end{array}$ & \multirow[t]{2}{*}{0.654} & 24 & $\begin{array}{l}0.79 \\
\pm 0.7\end{array}$ & \multirow[t]{2}{*}{0.009} & 24 & $\begin{array}{l}1.47 \\
\pm 0.7\end{array}$ & \multirow[t]{2}{*}{0.073} \\
\hline & Employed & 92 & $\begin{array}{l}0.94 \pm \\
0.6\end{array}$ & & 88 & $\begin{array}{l}0.51 \\
\pm 0.5\end{array}$ & & 85 & $\begin{array}{l}1.26 \\
\pm 0.8\end{array}$ & & 89 & $\begin{array}{l}1.82 \\
\pm 0.8\end{array}$ & \\
\hline \multirow[t]{2}{*}{ Income } & 10,000 and Below & 31 & $\begin{array}{l}0.75 \pm \\
0.7\end{array}$ & \multirow[t]{2}{*}{0.108} & 32 & $\begin{array}{l}0.55 \\
\pm 0.5\end{array}$ & 0.588 & 30 & $\begin{array}{l}1.25 \\
\pm 1.0\end{array}$ & 0.525 & 31 & $\begin{array}{l}1.54 \\
\pm 1.0\end{array}$ & 0.073 \\
\hline & Above 10,000 & 75 & $\begin{array}{l}0.97 \pm \\
0.6\end{array}$ & & 71 & $\begin{array}{l}0.49 \\
\pm 0.5\end{array}$ & & 69 & $\begin{array}{l}1.14 \\
\pm 0.7\end{array}$ & & 72 & $\begin{array}{l}1.86 \\
\pm 0.7\end{array}$ & \\
\hline Education Level & Primary and Below & 12 & $\begin{array}{l}1.40 \pm \\
0.7\end{array}$ & 0.001 & 12 & $\begin{array}{l}0.84 \\
\pm 0.6\end{array}$ & 0.085 & 12 & $\begin{array}{l}1.79 \\
\pm 0.8\end{array}$ & 0.014 & 12 & $\begin{array}{l}1.82 \\
\pm 0.8\end{array}$ & 0.979 \\
\hline & Secondary & 30 & $\begin{array}{l}0.66 \pm \\
0.6\end{array}$ & & 31 & $\begin{array}{l}0.56 \\
\pm 0.6\end{array}$ & & 29 & $\begin{array}{l}1.03 \\
\pm 0.9\end{array}$ & & 30 & $\begin{array}{l}1.76 \\
\pm 0.9\end{array}$ & \\
\hline & Post-Secondary & 68 & $\begin{array}{l}0.86 \pm \\
0.5\end{array}$ & & 66 & $\begin{array}{l}0.47 \\
\pm 0.5\end{array}$ & & 64 & $\begin{array}{l}1.16 \\
\pm 0.7\end{array}$ & & 65 & $\begin{array}{l}1.77 \\
\pm 0.7\end{array}$ & \\
\hline Number of Children & 3 and below & 83 & $\begin{array}{l}0.91 \pm \\
0.6\end{array}$ & 0.140 & 78 & $\begin{array}{l}0.54 \\
\pm 0.6\end{array}$ & 0.823 & 76 & $\begin{array}{l}1.16 \\
\pm 0.7\end{array}$ & 0.852 & 80 & $\begin{array}{l}1.87 \\
\pm 0.7\end{array}$ & 0.082 \\
\hline & Above 3 & 29 & $\begin{array}{l}0.71 \pm \\
0.5\end{array}$ & & 30 & $\begin{array}{l}0.51 \\
\pm 0.6\end{array}$ & & 28 & $\begin{array}{l}1.13 \\
\pm 1.0\end{array}$ & & 28 & $\begin{array}{l}1.55 \\
\pm 1.0\end{array}$ & \\
\hline $\begin{array}{l}\text { Age of Child(ren) with } \\
\text { Intellectual Disability }\end{array}$ & 9 and Below & 17 & $\begin{array}{l}0.73 \pm \\
0.8\end{array}$ & 0.242 & 15 & $\begin{array}{l}0.37 \\
\pm 0.4\end{array}$ & 0.091 & 15 & $\begin{array}{l}1.27 \\
\pm 0.9\end{array}$ & 0.029 & 15 & $\begin{array}{l}1.23 \\
\pm 0.9\end{array}$ & 0.000 \\
\hline & $10-12$ Years & 34 & $\begin{array}{l}0.97 \pm \\
0.6\end{array}$ & & 34 & $\begin{array}{l}0.73 \\
\pm 0.7\end{array}$ & & 34 & $\begin{array}{l}1.46 \\
\pm 0.9\end{array}$ & & 34 & $\begin{array}{l}2.14 \\
\pm 0.9\end{array}$ & \\
\hline & $13-18$ Years & 25 & $\begin{array}{l}1.03 \pm \\
0.7\end{array}$ & & 23 & $\begin{array}{l}0.47 \\
\pm 0.5\end{array}$ & & 23 & $\begin{array}{l}1.15 \\
\pm 0.6\end{array}$ & & 25 & $\begin{array}{l}1.97 \\
\pm 0.6\end{array}$ & \\
\hline & Over 18 Years & 38 & $\begin{array}{l}0.78 \pm \\
0.5\end{array}$ & & 39 & $\begin{array}{l}0.47 \\
\pm 0.4\end{array}$ & & 35 & $\begin{array}{l}0.90 \\
\pm 0.7\end{array}$ & & 37 & $\begin{array}{l}1.42 \\
\pm 0.7\end{array}$ & \\
\hline Number of People Living With & $1-3$ & 38 & $\begin{array}{l}0.93 \pm \\
0.7\end{array}$ & 0.805 & 36 & $\begin{array}{l}0.51 \\
\pm 0.5\end{array}$ & 0.941 & 34 & $\begin{array}{l}1.28 \\
\pm 0.7\end{array}$ & 0.394 & 36 & $\begin{array}{l}1.56 \\
\pm 0.7\end{array}$ & 0.055 \\
\hline & $4-6$ & 61 & $\begin{array}{l}0.86 \pm \\
0.6\end{array}$ & & 60 & $\begin{array}{l}0.55 \\
\pm 0.6\end{array}$ & & 59 & $\begin{array}{l}1.15 \\
\pm 0.8\end{array}$ & & 60 & $\begin{array}{l}1.91 \\
\pm 0.8\end{array}$ & \\
\hline & $6+$ & 14 & $\begin{array}{l}0.82 \pm \\
0.7\end{array}$ & & 14 & $\begin{array}{l}0.50 \\
\pm 0.6\end{array}$ & & 13 & $\begin{array}{l}0.92 \\
\pm 1.1\end{array}$ & & 14 & $\begin{array}{l}1.44 \\
\pm 1.1\end{array}$ & \\
\hline Marital Satisfaction & None & 48 & $\begin{array}{l}0.76 \pm \\
0.6\end{array}$ & 0.123 & 49 & $\begin{array}{l}0.41 \\
\pm 0.5\end{array}$ & 0.064 & 47 & $\begin{array}{l}1.05 \\
\pm 0.8\end{array}$ & 0.234 & 48 & $\begin{array}{l}1.81 \\
\pm 0.8\end{array}$ & 0.634 \\
\hline
\end{tabular}




\begin{tabular}{|c|c|c|c|c|c|c|c|c|c|c|c|c|c|}
\hline & Yes & 66 & $\begin{array}{l}0.94 \pm \\
0.7\end{array}$ & & 61 & $\begin{array}{l}0.60 \\
\pm 0.5\end{array}$ & & 59 & $\begin{array}{l}1.24 \\
\pm 0.8\end{array}$ & & 62 & $\begin{array}{l}1.73 \\
\pm 0.8\end{array}$ & \\
\hline \multirow[t]{2}{*}{ Marital Happiness } & None & 68 & $\begin{array}{l}0.95 \pm \\
0.7\end{array}$ & \multirow[t]{2}{*}{0.042} & 64 & $\begin{array}{l}0.57 \\
\pm 0.5\end{array}$ & \multirow[t]{2}{*}{0.116} & 61 & $\begin{array}{l}1.21 \\
\pm 0.8\end{array}$ & \multirow[t]{2}{*}{0.227} & 64 & $\begin{array}{l}1.75 \\
\pm 0.8\end{array}$ & \multirow[t]{2}{*}{0.993} \\
\hline & Yes & 45 & $\begin{array}{l}0.70 \pm \\
0.6\end{array}$ & & 45 & $\begin{array}{l}0.41 \\
\pm 0.5\end{array}$ & & 44 & $\begin{array}{l}1.02 \\
\pm 0.8\end{array}$ & & 45 & $\begin{array}{l}1.75 \\
\pm 0.8\end{array}$ & \\
\hline \multirow[t]{2}{*}{ Care Burden } & Yes & 96 & $\begin{array}{l}0.90 \pm \\
0.6\end{array}$ & \multirow[t]{2}{*}{0.187} & 94 & $\begin{array}{l}0.54 \\
\pm 0.6\end{array}$ & \multirow[t]{2}{*}{0.356} & 90 & $\begin{array}{l}1.28 \\
\pm 0.7\end{array}$ & \multirow[t]{2}{*}{0.000} & 93 & $\begin{array}{l}1.83 \\
\pm 0.7\end{array}$ & \multirow[t]{2}{*}{0.002} \\
\hline & No & 18 & $\begin{array}{l}0.69 \pm \\
0.6\end{array}$ & & 16 & $\begin{array}{l}0.41 \\
\pm 0.5\end{array}$ & & 16 & $\begin{array}{l}0.53 \\
\pm 0.7\end{array}$ & & 17 & $\begin{array}{l}1.14 \\
\pm 0.7\end{array}$ & \\
\hline \multirow[t]{2}{*}{ Depression } & Depression & 28 & $\begin{array}{l}1.04 \pm \\
0.8\end{array}$ & \multirow[t]{2}{*}{0.089} & 27 & $\begin{array}{l}0.45 \\
\pm 0.4\end{array}$ & \multirow[t]{2}{*}{0.392} & 26 & $\begin{array}{l}1.21 \\
\pm 0.6\end{array}$ & \multirow[t]{2}{*}{0.709} & 28 & $\begin{array}{l}1.47 \\
\pm 0.6\end{array}$ & \multirow[t]{2}{*}{0.048} \\
\hline & No depression & 89 & $\begin{array}{l}0.81 \pm \\
0.5\end{array}$ & & 86 & $\begin{array}{l}0.55 \\
\pm 0.6\end{array}$ & & 83 & $\begin{array}{l}1.14 \\
\pm 0.8\end{array}$ & & 85 & $\begin{array}{l}1.84 \\
\pm 0.8\end{array}$ & \\
\hline
\end{tabular}

Table 4 presents the results of multivariate analysis of factors that were significantly associated with the outcomes at the bivariate level. 
Multivariate analysis of factors associated with stigma

\begin{tabular}{|c|c|c|c|c|c|}
\hline \multirow[t]{2}{*}{ Variable } & \multirow[t]{2}{*}{ Category } & Unfair treatment & Stopping self & Overcoming stigma & $\begin{array}{l}\text { Positive } \\
\text { treatment }\end{array}$ \\
\hline & & $\beta(95 \%$ C.I) & $\beta(95 \%$ C.I) & $\beta(95 \%$ C.I) & $\beta(95 \%$ C.I) \\
\hline \multirow[t]{4}{*}{ Respondents' Age } & $18-25 y r s$ & $\mathrm{n} / \mathrm{s}$ & $\mathrm{n} / \mathrm{s}$ & $0.39(0.01 \text { to } 0.76)^{\star}$ & $\begin{array}{l}-0.24(-0.78 \text { to } \\
0.30)\end{array}$ \\
\hline & $26-35 y r s$ & $\mathrm{n} / \mathrm{s}$ & $\mathrm{n} / \mathrm{s}$ & $\begin{array}{l}-0.62(-1.05 \text { to } \\
-0.18)^{\star \star}\end{array}$ & $\begin{array}{l}0.20(-0.32 \text { to } \\
0.72)\end{array}$ \\
\hline & $36-45 y r s$ & $\mathrm{n} / \mathrm{s}$ & $\mathrm{n} / \mathrm{s}$ & $0.25(-0.07$ to 0.57$)$ & $\begin{array}{l}0.49(0.09 \text { to } \\
0.89)^{\star}\end{array}$ \\
\hline & $45+$ & $\mathrm{n} / \mathrm{s}$ & $\mathrm{n} / \mathrm{s}$ & Ref. & Ref. \\
\hline \multirow[t]{3}{*}{ Marital Status } & Single & $\begin{array}{l}-0.69(-1.18 \text { to } \\
-0.20)^{\star \star}\end{array}$ & $0.05(-0.40$ to 0.50$)$ & $-0.15(-0.70$ to 0.40$)$ & $\mathrm{n} / \mathrm{s}$ \\
\hline & Married & $\begin{array}{l}-0.55(-0.96 \text { to } \\
-0.13)^{\star \star}\end{array}$ & $\begin{array}{l}-0.14(-0.53 \text { to } \\
0.25)\end{array}$ & $\begin{array}{l}-0.42(-0.84 \text { to } \\
-0.01)^{\star}\end{array}$ & $\mathrm{n} / \mathrm{s}$ \\
\hline & Divorced/Separated & Ref. & Ref. & Ref. & $\mathrm{n} / \mathrm{s}$ \\
\hline \multirow[t]{2}{*}{ Employment Status } & Unemployed & $\begin{array}{l}-0.29(-0.54 \text { to } \\
-0.03)^{\star}\end{array}$ & $\mathrm{n} / \mathrm{s}$ & $\begin{array}{l}-0.61(-0.88 \text { to } \\
-0.33)^{\star \star \star}\end{array}$ & $\begin{array}{l}-0.04(-0.50 \text { to } \\
0.43)\end{array}$ \\
\hline & Employed & Ref. & $\mathrm{n} / \mathrm{s}$ & Ref. & Ref. \\
\hline \multirow[t]{2}{*}{ Income } & 10,000 and Below & $\mathrm{n} / \mathrm{s}$ & $\mathrm{n} / \mathrm{s}$ & $\mathrm{n} / \mathrm{s}$ & $\begin{array}{l}-0.04(-0.40 \text { to } \\
0.33)\end{array}$ \\
\hline & Above 10,000 & $\mathrm{n} / \mathrm{s}$ & $\mathrm{n} / \mathrm{s}$ & $\mathrm{n} / \mathrm{s}$ & Ref. \\
\hline \multirow[t]{3}{*}{ Education Level } & Primary and Below & $0.03(-0.44$ to 0.49$)$ & $0.43(0.00 \text { to } 0.86)^{*}$ & $0.53(0.04 \text { to } 1.03)^{\star}$ & $\mathrm{n} / \mathrm{s}$ \\
\hline & Secondary & $\begin{array}{l}-0.18(-0.43 \text { to } \\
0.07)\end{array}$ & $0.14(-0.09$ to 0.37$)$ & $0.13(-0.15$ to 0.41$)$ & $\mathrm{n} / \mathrm{s}$ \\
\hline & Post-Secondary & Ref. & Ref. & Ref. & $\mathrm{n} / \mathrm{s}$ \\
\hline \multirow[t]{2}{*}{ Number of Children } & 3 and below & $\mathrm{n} / \mathrm{s}$ & $\mathrm{n} / \mathrm{s}$ & $\mathrm{n} / \mathrm{s}$ & $\begin{array}{l}0.35(-0.11 \text { to } \\
0.80)\end{array}$ \\
\hline & Above 3 & $\mathrm{n} / \mathrm{s}$ & $\mathrm{n} / \mathrm{s}$ & $\mathrm{n} / \mathrm{s}$ & Ref. \\
\hline \multirow[t]{4}{*}{$\begin{array}{l}\text { Age of Child(ren) with Intellectual } \\
\text { Disability }\end{array}$} & 9 and Below & $\mathrm{n} / \mathrm{s}$ & $\begin{array}{l}-0.11(-0.40 \text { to } \\
0.19)\end{array}$ & $0.27(-0.09$ to 0.63$)$ & $\begin{array}{l}-0.24(-0.73 \text { to } \\
0.25)\end{array}$ \\
\hline & $10-12$ Years & $\mathrm{n} / \mathrm{s}$ & $0.14(-0.11$ to 0.39$)$ & $\begin{array}{l}0.47(0.11 \text { to } \\
0.82)^{\star \star}\end{array}$ & $\begin{array}{l}0.52(0.09 \text { to } \\
0.96)^{\star}\end{array}$ \\
\hline & $13-18$ Years & $\mathrm{n} / \mathrm{s}$ & $\begin{array}{l}-0.16(-0.43 \text { to } \\
0.11)\end{array}$ & $-0.09(-0.49$ to 0.31$)$ & $\begin{array}{l}0.25(-0.24 \text { to } \\
0.75)\end{array}$ \\
\hline & Over 18 Years & $\mathrm{n} / \mathrm{s}$ & Ref. & Ref. & Ref. \\
\hline \multirow[t]{3}{*}{ Number of People Living With } & $1-3$ & $\mathrm{n} / \mathrm{s}$ & $\mathrm{n} / \mathrm{s}$ & $\mathrm{n} / \mathrm{s}$ & $\begin{array}{l}-0.17(-0.78 \text { to } \\
0.43)\end{array}$ \\
\hline & $4-6$ & $\mathrm{n} / \mathrm{s}$ & $\mathrm{n} / \mathrm{s}$ & $\mathrm{n} / \mathrm{s}$ & $\begin{array}{l}0.22(-0.33 \text { to } \\
0.77)\end{array}$ \\
\hline & $6+$ & $\mathrm{n} / \mathrm{s}$ & $\mathrm{n} / \mathrm{s}$ & $\mathrm{n} / \mathrm{s}$ & Ref. \\
\hline \multirow[t]{2}{*}{ Marital Satisfaction } & None & $\mathrm{n} / \mathrm{s}$ & $\begin{array}{l}-0.03(-0.43 \text { to } \\
0.37)\end{array}$ & $\mathrm{n} / \mathrm{s}$ & $\mathrm{n} / \mathrm{s}$ \\
\hline & Yes & $\mathrm{n} / \mathrm{s}$ & Ref. & $\mathrm{n} / \mathrm{s}$ & $\mathrm{n} / \mathrm{s}$ \\
\hline \multirow[t]{2}{*}{ Marital Happiness } & None & $0.11(-0.12$ to 0.34$)$ & $\begin{array}{l}-0.01(-0.39 \text { to } \\
0.38)\end{array}$ & $\mathrm{n} / \mathrm{s}$ & $\mathrm{n} / \mathrm{s}$ \\
\hline & Yes & Ref. & Ref. & $\mathrm{n} / \mathrm{s}$ & $\mathrm{n} / \mathrm{s}$ \\
\hline \multirow[t]{2}{*}{ Care Burden } & Yes & $\mathrm{n} / \mathrm{s}$ & $\mathrm{n} / \mathrm{s}$ & $\begin{array}{l}0.65(0.32 \text { to } \\
0.98)^{\star \star \star}\end{array}$ & $\begin{array}{l}0.59(0.20 \text { to } \\
0.97)^{\star \star}\end{array}$ \\
\hline & No & $\mathrm{n} / \mathrm{s}$ & $\mathrm{n} / \mathrm{s}$ & Ref. & Ref. \\
\hline
\end{tabular}




\begin{tabular}{|c|c|c|c|c|c|}
\hline \multirow[t]{2}{*}{ Variable } & \multirow[t]{2}{*}{ Category } & Unfair treatment & Stopping self & Overcoming stigma & $\begin{array}{l}\text { Positive } \\
\text { treatment }\end{array}$ \\
\hline & & $\beta(95 \%$ C.I) & $\beta(95 \%$ C.I) & $\beta(95 \%$ C.I) & $\beta(95 \%$ C.I) \\
\hline Depression & BDI & $0.00(-0.01$ to 0.02$)$ & $\begin{array}{l}-0.02(-0.03 \text { to } \\
-0.01)^{\star \star}\end{array}$ & $\begin{array}{l}-0.02(-0.04 \text { to } \\
-0.01)^{\star *}\end{array}$ & $\begin{array}{l}-0.01(-0.02 \text { to } \\
0.01)\end{array}$ \\
\hline
\end{tabular}

\section{Unfair treatment sub-scale}

Participants who were single and married had significantly lower scores as compared to those who were either divorced or separated. Those who were unemployed had significantly lower scores as compared to those who were employed. No significant associations were found between unfair treatment and depression scores.

\section{Stopping self}

Participants with primary and below levels of education had significantly high scores as compared to those with post-secondary education. High scores of stopping self were associated with significantly low depression scores.

\section{Overcoming stigma}

Participants who were in the younger age band (18-25 years) had significantly higher scores as compared to those who were old (45 + years), however participants who were ages 26-35 years had significantly lower scores as compared to those aged 45 and above years. Those who were married had significantly low scores as compared to those who were either divorced or separated. Those who were unemployed had significantly lower scores as compared to those who were employed. Participants with children with intellectual disability ages 10-12 years had significantly high scores as compared to those with children over 18 years. Having a care burden was associated with high scores as compared to those without. High scores were negatively associated with higher depression scores.

\section{Positive treatment}

Participants ages 36-45 years had significantly high scores as compared to those aged above 45 years. Respondents with children with intellectual disability aged 10-12 years had significantly higher scores as compared to those with children with intellectual disability aged over 18 years. Having a care burden was associated with high scores as compared to those without. No significant associations were found between positive treatment scores and depression scores.

\section{Discussion}

The study explores the mental health needs of caregivers of children with intellectual disabilities face and leads us to conclude that they is a need to routinely screen caregivers of children with intellectual disability for depressive symptoms. In this study $41.3 \%$ of parents met the criterion for being at risk of clinical depression (using a score of 11 or higher using BDI scale). A previous study carried out in Kenya demonstrated a higher prevalence at $79 \%$ of caregiver depressive symptoms (Mbugua et al., 2011). This particular study looked at prevalence of depression among caregivers of children with intellectual disability in a rural setting in Kenya. In our study, caregivers lived and worked in various parts of Kenya and their children are registered in Special public primary schools in Nairobi County.

The schools we selected are boarding schools thus the parents are not primary caregivers during the term and only take care of the children during the school holidays. Unlike most day schools where the parent frequently interacts with the child the children in the study were in boarding schools and only had minimal interactions with parents during the school holidays and special school dates such as visiting or parents meetings. There lies the significance of such a study that addresses a diverse sample of caregivers, assessing their mental health needs and challenges.

We found an association between gender and a higher risk of depression. There were more female than male caregivers because most female parents were the sole caregivers of the children and were also emotionally attached to their children unlike their male counterparts. The study was carried out during school meetings which we learnt were mostly attended to by female caregivers to check on their children. Most of the parents were married (69.19\%), employed (78.51\%) and had significant incomes to support burden of care of their children.

The current study found out that younger parents (18-26 years) were at a higher risk of depression (60\%). In support of these findings was a study by(Dave et al., 2014), carried out in Gujarat, India study which found that being a younger caregiver was predictive of higher levels of depressive symptoms.

There was a statistically significant association between marital status and depression $(P=0.003)$. The divorced and separated parents had significant higher levels of depression (76.47\%) than the single and the married ones. In an earlier study, (Frey, Fewell, Vadasy, \& Greenberg, 1989) found that 16.5\% of divorced/separated and single caregivers may have adopted coping strategies therefore had a lower risk of clinical depression compared to married caregiver. In the current study, married caregivers were the majority (69.91\%) and cited to have marital satisfaction and happiness thus lower depression scores. By contrast, (Mbugua et al., 2011) found that single caregivers were at higher risk of depression due to lack of emotional support from the spouse).

Page $10 / 14$ 
There is need to avail a support system to prevent or reduce the risk of depression. This may include specialized information, caregiver training, education and family counselling aimed at a more holistic way of care.

The other statistically significant risk factor to the development of depressive symptoms was caregiver burden with a $\mathrm{p}$ value of 0.033 . In the current study, the results showed that $38.36 \%(n=47)$ experienced burden of care. (Saunders, 2003)found that caring exerts pressure on the caregiver. As the stressors increase and the condition persist, the caregiver symptoms may worsen. This deterioration of caregiver health may impact on their ability to maintain their various roles and responsibilities.

This study also found statistically significant relationship between caregiver education and depressive symptoms $p=<0.001$. Most of the parents $61.06 \%$ $(n=69)$ had post-secondary education, $27.43 \%(n=31)$ had secondary education while $9.73 \%(n=11)$ were primary school graduates and others had dropped out at primary level. Of all the caregivers only $1.77 \%(n=2)$ did not have any formal education. Of those with primary education $(n=11) 63.64 \%$ had depressive symptoms. This finding is in contrast with (Grote et al., 2010; McLearn, Minkovitz, Strobino, Marks, \& Hou, 2006; Mclennan, Kotelchuck, \& Cho, 2001)undertaken in Florida a city in the first world unlike Kenya a developing country found that caregivers who had lower levels of education were more likely to report depressive symptoms. Higher level of education was associated with employment (business or self-employment), higher levels of income and lower risks of depressive symptoms.

(Thornicroft, Brohan, Rose, Sartorius, \& Leese, 2009)in their study found that people with mental health problems experienced stigma and discrimination frequently. This is in keeping with the current study which found that $36.36 \%$ of caregivers experienced discrimination by family members, friends and the public. Fifteen-point seven percent (15.7\%) had stopped themselves from applying for work, education and training. However, the rates were lower than the study by (Evans-Lacko, Corker, Williams, Henderson, \& Thornicroft, 2014) as the participants had not been previously diagnosed with depression.

Ali et al., (2012) explored the role of stigma in parental wellbeing and this study demonstrated that stigma is associated with caregivers' burden, marital satisfaction and experiences of social exclusion in parents of children with intellectual disabilities. The current study found stigma and discrimination to be significantly related to parent's depressive symptoms independently of other variables. In the study stigma reflects the extent to which parents felt fairly untreated, self-stigma, their ability to avoid stigma and areas of positive treatment in regards to the disability of their child. These study findings are in keeping with a study done on global patterns of experienced and anticipated discrimination (Lasalvia et al., 2013).

Higher levels of experienced discrimination were associated with greater levels of depression. Similar studies found that levels of discrimination were high in depressed persons (Corker et al., 2013; Lasalvia et al., 2013; Thornicroft et al., 2009)

In the DISC-12 anticipated discrimination occurs when a person limits their own involvement in important aspects of everyday life because of the expectation of being discriminated against (for example, when an individual does not apply for a job because he/she fully expects to fail in any such application) (Lasalvia et al., 2013). A study by (Evans-Lacko et al., 2014)showed higher levels of experienced discrimination in a population diagnosed with depression.

Stigma and discrimination cut across all demographic factors with most parents feeling unfairly treated, this could be due to society stigma towards disability as well as lack of resources in our country to provide better services to people living with disabilities. These results are consistent with (Seltzer, Greenberg, Floyd, Pettee, \& Hong, 2001)study on the life course impacts of parenting a child with a disability which found that there are emotional burdens associated with the stigma of the disabilities.

The results of this study show a significant association between depressive symptoms and unfair treatment. This is in keeping with a study done in Ireland that showed that parents of children with disabilities reported more depressive symptomology, higher stigma and lower self-esteem Inability to overcome stigma significantly increased levels of depression,(Cantwell et al., 2015).

Higher levels of anticipated discrimination were moderately associated with increasing age, but the strongest association was with gender. Females anticipated higher levels of discrimination than males in several areas including housing, employment and family life. The introduction of methods to minimize discrimination towards people with intellectual disabilities at individual, institutional, and structural levels and the identification of effective strategies to reduce anticipated discrimination by people with intellectual disabilities towards themselves might be necessary to tackle stigma and discrimination.

Providing parents with methods for dealing with perceived stigma and highlighting the importance of attending support groups for emotional support may mitigate some of the negative consequences of stigma, such as the impact on coping and support.

Overall, $15.7 \%$ of participant felt that they had stopped themselves from initiating a close relationship, applying for work and from applying for education or training. This is consistent with global study (Lasalvia et al., 2013) that reported 37\% of participants had stopped themselves from initiating a close personal relationship using the same scale. Importantly $72.73 \%$ reported positive treatment in various domains of life. These findings suggest that the social environment could be a source of support to caregivers, depending on the context or the personal resources of the individual.

\section{Limitations Of The Study}

The study did not look into pre-existing depression which may have had an influence on the findings. The study was limited to urban schools, and thus the results cannot be generalized to the rural population. It is important to note that the participants selected for this study were drawn from a convenience

Page $11 / 14$ 
sample of caregivers whose children were registered in special schools in Nairobi which are few. The sample has a higher level of formal education and a greater percentage is married. The results may not reflect the general population of parents of children with intellectual disabilities.

Larger populations randomly selected from a national sample may be the focus in future studies to determine with more confidence the prevalence of depression and levels of stigma among caregivers of children with intellectual disability.

\section{Conclusions}

Stigma and discrimination acts as a barrier to social participation and successful vocational participation for parents and in turn a contributor to development of depressive symptoms. The rates of parent's depressive symptoms and levels of stigmatization suggest that interventions should include attention to the mental health and recovery of parents in addition to their children. Young parents, male parents and those with lower levels of education are particularly at risk of depression and high levels of discrimination and stigma. The findings suggest that future interventions designed to decrease stigma in society should include parents of children with disabilities.

\section{Declarations}

\section{Competing interests}

The authors declare that they have no competing interests.

\section{Authors' contributions}

The study was carried out by Mercy Githara who drafted the concept, collected data and carried out initial analyses of her survey. Manasi Kumar was her primary mentor and helped in conceptualization, writing up and conducting multivariate analysis. Anne Obondo was her second supervisor who assisted during planning of the research concept and reviewed the MS. Graham Thornicroft provided technical guidance with the use of stigma tool and read and commented on the manuscript. Albert assisted with statistical analyses.

\section{Ethical approval and consent to participate}

The University of Nairobi/Kenyatta National Hospital Ethics Review Committee provided ethical approval for this study (P100/02/2015). All patients included in this study provided informed consent prior to participation in the study and gave consent for publication of the findings. Participants gave written informed consent and consented to their findings being published.

\section{Acknowledgements}

We would like to thank our study participants, numerous caregivers and school personnel who supported data collection.

MK would like to acknowledge the support of NIMH U19 focusing on Strengthening mental health research in Africa focusing on Uganda, Kenya, Ghana and South Africa (SMART-Africa; U19 MH110001-01) and Fogarty Foundation/NIH K43 study on Implementing mental health interventions for pregnant adolescents in LMICs' (TW010716-01A1).

GT is supported by the National Institute for Health Research (NIHR) Applied Research Collaboration South London at King's College London NHS Foundation Trust, and by the NIHR Asset Global Health Unit award. The views expressed are those of the author(s) and not necessarily those of the NHS, the NIHR or the Department of Health and Social Care. GT also receives support from the National Institute of Mental Health of the National Institutes of Health under award number R01MH100470 (Cobalt study). GT is supported by the UK Medical Research Council in relation the Emilia (MR/S001255/1) and Indigo Partnership (MR/R023697/1) awards.

\section{Consent to publish}

Informed consent was given from participants in this research for future uses of data, such as publication, preservation and long-term use of research data. Confidentiality was assured. The information collected was kept confident, serial numbers was used instead of names.

\section{Availability of materials}

Deidentified data file would be made available upon reasonable request to the corresponding author.

\section{Funding}

This research was primarily funded by the first author who carried out data collection as part of her Masters in Clinical psychology program.

\section{References}

1. Aaron Resch, J., Elliott, T. R., \& Benz, M. R. (2012). Depression among parents of children with disabilities. Families, Systems and Health. https://doi.org/10.1037/a0030366

Page $12 / 14$ 
2. Ali, A., Hassiotis, A., Strydom, A., \& King, M. (2012). Self stigma in people with intellectual disabilities and courtesy stigma in family carers: A systematic review. Research in Developmental Disabilities, 33(6), 2122-2140. https://doi.org/10.1016/j.ridd.2012.06.013

3. Beck, A., Steer, R. A., \& Brown, G. (1996). Beck Depression Inventory-II.

4. Bourke-Taylor, H., Pallant, J. F., Law, M., \& Howie, L. (2012). Predicting mental health among mothers of school-aged children with developmental disabilities: The relative contribution of child, maternal and environmental factors. Research in Developmental Disabilities, 33(6), 1732-1740. https://doi.org/10.1016/j.ridd.2012.04.011

5. Britto, P. R., Lye, S. J., Proulx, K., Yousafzai, A. K., Matthews, S. G., Vaivada, T., ... Bhutta, Z. A. (2017). Nurturing care: promoting early childhood development. The Lancet, 389(10064), 91-102. https://doi.org/10.1016/S0140-6736(16)31390-3

6. Brohan, E., Clement, S., Rose, D., Sartorius, N., Slade, M., \& Thornicroft, G. (2013). Development and psychometric evaluation of the Discrimination and Stigma Scale (DISC). Psychiatry Research, 208(1), 33-40. https://doi.org/10.1016/j.psychres.2013.03.007

7. Burke, L. (2003). International Review of Psychiatry The impact of maternal depression on familial relationships The impact of maternal depression on familial relationships. International Review of Psychiatry. https://doi.org/10.1080/0954026031000136866

8. Cantwell, J., Muldoon, O., \& Gallagher, S. (2015). The influence of self-esteem and social support on the relationship between stigma and depressive symptomology in parents caring for children with intellectual disabilities. Journal of Intellectual Disability Research, 59(10), 948-957.

https://doi.org/10.1111/jir.12205

9. Corker, E., Hamilton, S., Henderson, C., Weeks, C., Pinfold, V., Rose, D., ... Thornicroft, G. (2013). Experiences of discrimination among people using mental health services in England 2008-2011. British Journal of Psychiatry. https://doi.org/10.1192/bjp.bp.112.112912

10. Czaja, R., \& Blair, J. (2005). Designing surveys: a guide to decisions and procedures. Pine Forge Press.

11. Dave, D., Mittal, S., Tiwari, D., Parmar, M., Gedan, S., \& Patel, V. (2014). Study of Anxiety and Depression in Caregivers of Intellectually Disabled Children. Journal of Research in Medical and Dental Science, 2(1), 8. https://doi.org/10.5455/jrmds.2014212

12. Eisenhower, A. S., Baker, B. L., \& Blacher, J. (2005). Preschool children with intellectual disability: syndrome specificity, behaviour problems, and maternal well-being. Journal of Intellectual Disability Research, 49(9), 657-671. https://doi.org/10.1111/j.1365-2788.2005.00699.x

13. Estes, A., Olson, E., Sullivan, K., Greenson, J., Winter, J., Dawson, G., \& Munson, J. (2013). Parenting-related stress and psychological distress in mothers of toddlers with autism spectrum disorders. Brain \& Development, 35(2), 133-138. https://doi.org/10.1016/j.braindev.2012.10.004

14. Evans-Lacko, S., Corker, E., Williams, P., Henderson, C., \& Thornicroft, G. (2014). Effect of the Time to Change anti-stigma campaign on trends in mental-illness-related public stigma among the English population in 2003-13: an analysis of survey data. The Lancet Psychiatry, 1(2), 121-128. https://doi.org/10.1016/S2215-0366(14)70243-3

15. Frey, K. S., Fewell, R. R., Vadasy, P. F., \& Greenberg, M. T. (1989). Parental Adjustment and Changes in Child Outcome Among Families of Young Handicapped Children. Topics in Early Childhood Special Education. https://doi.org/10.1177/027112148900800404

16. Gallagher, S., \& Hannigan, A. (2014). Depression and chronic health conditions in parents of children with and without developmental disabilities: The growing up in Ireland cohort study. Research in Developmental Disabilities. https://doi.org/10.1016/j.ridd.2013.11.029

17. Ghassemzadeh, H., Mojtabai, R., Karamghadiri, N., \& Ebrahimkhani, N. (2005). Psychometric properties of a persian-language version of the Beck Depression Inventory- second edition: BDI-II Persian. Depression and Anxiety, 21(4), 185-192. https://doi.org/10.1002/da.20070

18. Goffman, E. (2009). Stigma: Notes on the management of spoiled identity. Retrieved from https://books.google.com/books? $\mathrm{hl}=$ en\&l $\mathrm{r}=\& \mathrm{id}=\mathrm{zuMFX}$ TMAqAC\&oi=fnd\&pg=PA1\&dq=Goffman,+E.+

(2009).+Stigma:+Notes+on+the+management+of+spoiled+identity.+Simon+and+Schuster\&ots=R91IKu2CKe\&sig=nFmWVTICDIb37FITam5_xWEGch4

19. Goffman, Erving. (1963). Stigma notes on the management of spoiled identity. Retrieved from https://www.worldcat.org/title/stigma-notes-on-themanagement-of-spoiled-identity/oclc/223060

20. Grote, N. K., Bridge, J. A., Gavin, A. R., Melville, J. L., lyengar, S., \& Katon, W. J. (2010). A meta-analysis of depression during pregnancy and the risk of preterm birth, low birth weight, and intrauterine growth restriction. Archives of General Psychiatry.

https://doi.org/10.1001/archgenpsychiatry.2010.111

21. Kojima, M., Furukawa, T. A., Takahashi, H., Kawai, M., Nagaya, T., \& Tokudome, S. (2002). Cross-cultural validation of the Beck Depression Inventory-II in Japan. Psychiatry Research. https://doi.org/10.1016/S0165-1781(02)00106-3

22. Lasalvia, A., Zoppei, S., Van Bortel, T., Bonetto, C., Cristofalo, D., Wahlbeck, K., ... Thornicroft, G. (2013). Global pattern of experienced and anticipated discrimination reported by people with major depressive disorder: a cross-sectional survey. The Lancet, 381(9860), 55-62.

https://doi.org/10.1016/S0140-6736(12)61379-8

23. Masulani-Mwale, C., Mathanga, D., Kauye, F., \& Gladstone, M. (2018). Psychosocial interventions for parents of children with intellectual disabilities-A narrative review and implications for low income settings. Mental Health and Prevention. https://doi.org/10.1016/j.mhp.2018.05.003

24. Masulani-Mwale, Charles, Kauye, F., Gladstone, M., \& Mathanga, D. (2019). Development of a psycho-social intervention for reducing psychological distress among parents of children with intellectual disabilities in Malawi. PLOS ONE, 14(2), e0210855. https://doi.org/10.1371/journal.pone.0210855

25. Mbugua, M. N., Kuria, M. W., \& Ndetei, D. M. (2011). The Prevalence of Depression among Family Caregivers of Children with Intellectual Disability in a Rural Setting in Kenya. International Journal of Family Medicine, 2011, 1-5. https://doi.org/10.1155/2011/534513

26. McLearn, K. T., Minkovitz, C. S., Strobino, D. M., Marks, E., \& Hou, W. (2006). Maternal depressive symptoms at 2 to 4 months post partum and early parenting practices. Archives of Pediatrics and Adolescent Medicine. https://doi.org/10.1001/archpedi.160.3.279 
27. MCLENNAN, J. D., KOTELCHUCK, M., \& CHO, H. (2001). Prevalence, Persistence, and Correlates of Depressive Symptoms in a National Sample of Mothers of Toddlers. Journal of the American Academy of Child \& Adolescent Psychiatry, 40(11), 1316-1323. https://doi.org/10.1097/00004583200111000-00012

28. Musyimi, C. W., Mutiso, V. N., Nayak, S. S., Ndetei, D. M., Henderson, D. C., \& Bunders, J. (2017). Quality of life of depressed and suicidal patients seeking services from traditional and faith healers in rural Kenya. Health and Quality of Life Outcomes. https://doi.org/10.1186/s12955-017-0657-1

29. Ndetei, D. M., Khasakhala, L. I., Kuria, M. W., Mutiso, V. N., Ongecha-Owuor, F. A., \& Kokonya, D. A. (2009). The prevalence of mental disorders in adults in different level general medical facilities in Kenya: A cross-sectional study. Annals of General Psychiatry. https://doi.org/10.1186/1744-859X-8-1

30. Neece, C., \& Baker, B. (2008). Predicting maternal parenting stress in middle childhood: the roles of child intellectual status, behaviour problems and social skills. Journal of Intellectual Disability Research: JIDR, 52(12), 1114-1128. https://doi.org/10.1111/j.1365-2788.2008.01071.x

31. Owalabi Bakari, M., Agomoh, A. O., Ebigbo, P. O., Eaton, J., Okonkwo, K. O., Onwukwe, J. U., \& Onyeama, G. M. (2009). Etiological explanation, treatability and preventability of childhood autism: A survey of Nigerian healthcare workers' opinion. Annals of General Psychiatry. https://doi.org/10.1186/1744-859X-8-6

32. Saunders, J. C. (2003). Families Living with Severe Mental Illness: A Literature Review. Issues in Mental Health Nursing, 24(2), 175-198. https://doi.org/10.1080/01612840305301

33. Seltzer, M. M., Greenberg, J. S., Floyd, F. J., Pettee, Y., \& Hong, J. (2001). Life course impacts of parenting a child with a disability. American Journal on Mental Retardation. https://doi.org/10.1352/0895-8017(2001)106<0265:LCIOPA>2.0.CO;2

34. Singer, G. H. S. (2006). Meta-analysis of comparative studies of depression in mothers of children with and without developmental disabilities. American Journal on Mental Retardation. https://doi.org/10.1352/0895-8017(2006)111[155:MOCSOD]2.0.C0;2

35. Thornicroft, G., Brohan, E., Rose, D., Sartorius, N., \& Leese, M. (2009). Global pattern of experienced and anticipated discrimination against people with schizophrenia: a cross-sectional survey. The Lancet. https://doi.org/10.1016/S0140-6736(08)61817-6

36. Thornicroft, G., Rose, D., Kassam, A., \& Sartorius, N. (2007). Stigma: ignorance, prejudice or discrimination? British Journal of Psychiatry, 190(3), 192193. https://doi.org/10.1192/bjp.bp.106.025791

37. Tilahun, D., Hanlon, C., Fekadu, A., Tekola, B., Baheretibeb, Y., \& Hoekstra, R. A. (2016). Stigma, explanatory models and unmet needs of caregivers of children with developmental disorders in a low-income African country: a cross-sectional facility-based survey. BMC Health Services Research, 16(1), 152. https://doi.org/10.1186/s12913-016-1383-9

38. van der Mark, E. J., Conradie, I., Dedding, C. W. M., \& Broerse, J. E. W. (2019). Exploring adaptation and agency of mothers caring for disabled children in an urban settlement in South Africa: A qualitative study. Women's Studies International Forum. https://doi.org/10.1016/j.wsif.2019.102271

39. WHO. (2011). World Report on Disability 2011. American Journal of Physical Medicine Rehabilitation Association of Academic Physiatrists, 91 , 549. https://doi.org/10.1136/ip.2007.018143 